P. K. T. LO, M. C. WILLIS* (UNIVERSITY OF OXFORD, UK)

Nickel(II)-Catalyzed Addition of Aryl and Hereoaryl Boroxines to the Sulfinylamine Reagent TrNSO: The Catalytic Synthesis of

Sulfinamides, Sulfonimidamides, and Primary Sulfonamides

J. Am. Chem. Soc. 2021, 143, 15576-15581, DOI: 10.1021/jacs.1c08052.

\title{
Synthesis of Sulfinamides, Sulfonimidamides, and Sulfonamides: A One-Pot Procedure to Rule them All
}

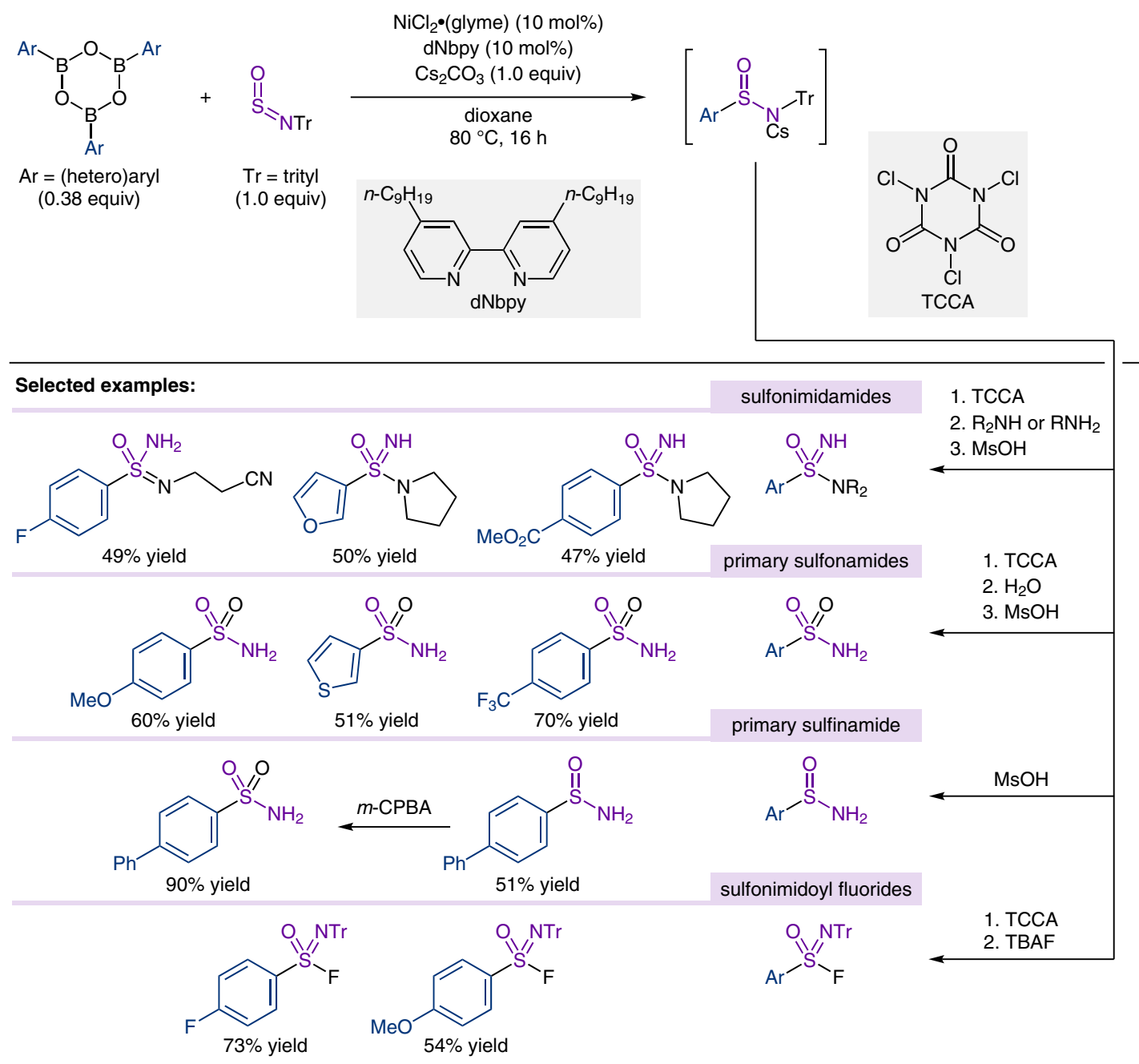

Significance: A one-pot approach for the synthesis of a variety of sulfinamides and derivatives is presented. Employing a commercially available catalyst and ligand, the catalyst loading can be lowered to $1 \mathrm{~mol} \%$ for large-scale reactions. Trichloroisocyanuric acid (TCCA) is used as a mild, inexpensive, and safe chlorination agent.

\section{Category}

Metals in Synthesis

\section{Key words}

boroxines

nickel catalysis

sulfinylamines

sulfonimidamides

trichloroisocyanuric acid
Comment: Boroxines are used as an alternative to moisture- and air-sensitive organometallic reagents, which often exclude a wide range of functional groups. Sulfonimidamides, primary sulfonamides, primary sulfinamides, and sulfonimidoyl fluorides are all obtained in moderate to good yields. 